\title{
CURVAS DE CRESCIMENTO DE FÊMEAS DA RAÇA NELORE E SEUS CRUZAMENTOS
}

\author{
Growth curves of female nelore and their crosses
}

\author{
Tarcisio de Moraes Gonçalves 1 , Marco Aurélio Dessimoni Dias², Jairo Azevedo Junior², \\ Mary Ana Petersen Rodriguez ${ }^{3}$, Vivian Dagnesi Timpani ${ }^{2}$, Antônio Ilson Gomes de Oliveira ${ }^{2}$
}

\begin{abstract}
RESUMO
Seleção para características do crescimento pode ser feita após ajustadas funções de crescimento para todos os animais sob seleção. Porém, a curva de crescimento de fêmeas é influenciada pelas gestações e lactações subsequentes. Neste trabalho, métodos são descritos para selecionar uma função que ajuste a curva de crescimento de fêmeas bovinas considerando-se os efeitos de variação de peso corporal devido a gestação e a lactação. Foram utilizados dados provenientes do Instituto Agronômico do Paraná, constituindo-se de 15.271 observações de pesos de 103 fêmeas, sendo 43 Nelore, $10 \mathrm{~F}_{1}$ Guzerá x Nelore, $29 \mathrm{~F}_{1}$ Red Angus x Nelore, $5 \mathrm{~F}_{1}$ Marchigiana x Nelore e $16 \mathrm{~F}_{1}$ Simental x Nelore. Comparou-se o ajuste das funções de Brody, Gompertz, Logística, Von Bertalanffy e Michaelis-Menten reparametrizada (MMr) aos efeitos do ganho de peso em função da idade, sendo adicionado a todas as funções o ganho de peso durante a gestação e a perda de peso na lactação de fêmeas da raça Nelore e Cruzadas. Ao ajustar as funções, observou-se um Coeficiente de Determinação Múltiplo Ajustado variando entre 0,7552 e 0,9267. Observou-se o menor Quadrado Médio do Resíduo e o Desvio Médio Absoluto para a função de MMr. Para descrição da curva de crescimento em função da idade das fêmeas, deve ser ajustada a função de MMr.
\end{abstract}

Termos para indexação: Crescimento de fêmeas, idade, gestação, lactação.

\begin{abstract}
Selection for growth characteristics can be done after fitting growth functions for all breeding animals. However, in the female population the growth curve is influenced by gestation and subsequent lactations. In this work methods are described to select a function that fits a growth curve of bovine females considering the varying effects of body weight due to gestation and lactation. Data from the Paraná Agronomic Institute (IAPAR) were used consisting of 15.271 weight observations of 103 females distributed as 43 Nelore, 10 F1 Guzerá x Nelore, 29 F1 Red Angus x Nelore, 5 F1 Marchigiana $\mathrm{x}$ Nelore and 16 F1 Simental x Nelore. The function adjustment of Brody, Gompertz, Logística, Von Bertalanffy and Michaelis-Menten reparametrized MMr were compared to the effects of weight gain as a function of age, adding to all functions the weight gain during gestation and the weight loss in the lactation of Nelore and crossbreed females. It was observed an Adjusted Coefficient of Multiple Determination varying from 0.7552 to 0.9267 . Lower Residue Mean Square and Absolute Mean Deviation were observed for the MMr function. The MMr function must be adjusted in order to describe the growth curve as a function of the female age.
\end{abstract}

Index terms: Growing females, age, gestation, lactation.

(Recebido em 9 de fevereiro de 2010 e aprovado em 11 de novembro de 2010)

\section{INTRODUÇÃO}

A produção de carne em rebanhos bovinos é influenciada, em maior ou menor grau, pelo genótipo do animal associado às condições de ambiente ao qual é submetido. Nessa espécie, a maioria das pesquisas sobre crescimento é voltada para as etapas iniciais do crescimento, limitando-se às medidas de idades-padrão como peso e a desmama, ao ano e ao sobreano, o que geralmente resulta em aumento do tamanho dos animais adultos, elevando custos de manutenção das vacas no rebanho e reduzindo vantagens econômicas do aumento de peso dos animais ao abate (Garnero et al., 2004).

O sucesso da atividade é determinado por um controle estratégico da produção, sendo necessário descrever o crescimento animal, uma vez que todos os processos produtivos estão diretamente relacionados ao crescimento. Normalmente essa descrição é feita por meio de curvas em que se relaciona o peso do animal com a sua idade.

Uma função para descrever o crescimento é considerada apropriada quando resume as informações dos pesos dos animais em um pequeno conjunto de

\footnotetext{
${ }^{1}$ Universidade Federal de Lavras/UFLA - Departamento de Zootecnia/DZO - Cx. P. 3037 - 37200-000 - Lavras, MG - tarcisio@dzo.ufla.br ${ }^{2}$ Universidade Federal de Lavras/UFLA - Departamento de Zootecnia/DZO - Lavras, MG

${ }^{3}$ Universidade de São Paulo/USP - Escola Superior de Agricultura Luiz de Queiroz/ESALQ - Piracicaba, SP
} 
parâmetros que podem ser interpretados biologicamente (López et al., 2000) e usados para derivar outras características relevantes do crescimento animal. Sendo interpretadas biologicamente, essas características podem ser utilizadas em programas de melhoramento buscando o aumento da eficiência econômica e biológica do rebanho. Alguns trabalhos sugerem que as variáveis da curva de crescimento são herdáveis em diferentes espécies, e isso torna possível a mudança na forma da curva de crescimento através da seleção com base nessas características (Lambe et al., 2006).

Vários autores ajustam funções de regressão linear, pois possuem aplicação em diversas áreas do conhecimento e descrevem com facilidade o relacionamento entre variáveis. Entretanto, quando se deseja descrever os processos biológicos, essas funções não permitem um bom ajuste. Dessa forma, propõe-se cada vez mais a utilização de funções não-lineares para descrever o crescimento.

As funções não-lineares se ajustam melhor às diferentes informações relacionadas a peso-idade durante o crescimento, permitindo o agrupamento dessas informações em poucos parâmetros biologicamente interpretáveis (como, por exemplo, taxa de crescimento e declividade da curva de lactação) que facilitam o entendimento do fenômeno (Brody, 1945).

Algumas funções não-lineares foram pouco testadas para descrever o crescimento de bovinos como a de Bridges e a de Michaelis-Menten reparametrizada. Ambas têm sido utilizadas para descrever o crescimento em espécies domésticas. Entretanto, o ajuste dessas funções de crescimento para diversas espécies tende a ser semelhante ou pior do que as funções de Richards ou Gompertz (Lambe et al., 2006).

A função de Michaelis-Menten reparametrizada é flexível para descrever o comportamento sigmoidal da curva de crescimento animal. Os parâmetros dessa função não-linear também possuem interpretação biológica e podem ser utilizados para calcular as estimativas das características de crescimento, como a taxa de crescimento pós-natal. A qualidade de ajuste dessa função é semelhante à de Richards (1959), e ambas foram ligeiramente superiores às de Gompertz. Por isso, a função de Michaelis-Menten, reparametrizada com o seu ponto de inflexão variável, descreve adequadamente o crescimento em uma ampla variedade de animais (López et al., 2000).

Objetivou-se, com este trabalho, comparar o ajuste das funções de Brody, Gompertz, Logística, Von Bertalanffy e Michaelis-Menten reparametrizada ao ganho de peso em função da idade, ajustando, simultaneamente, o ganho de peso durante a gestação e a perda de peso na lactação, em fêmeas da raça Nelore e Cruzadas.

\section{MATERIAL E MÉTODOS}

Foram utilizados 15.271 registros de pesos de 103 fêmeas, sendo: 43 Nelore (N), 10 F 1 Guzerá x Nelore (G), 29 $\mathrm{F}_{1}$ Red Angus x Nelore (R), $5 \mathrm{~F}_{1}$ Marchigiana x Nelore (M) e $16 \mathrm{~F}_{1}$ Simental x Nelore (S) provenientes do Instituto Agronômico do Paraná (IAPAR), Estação Experimental de Paranavaí - região noroeste do estado do Paraná.

Os dados foram coletados de seis em seis meses do nascimento à primeira cobertura e pesados aproximadamente a cada 30 dias, a partir do primeiro parto, com a última pesagem ocorrendo, no mínimo, após 40 meses de idade e peso vivo superior a $400 \mathrm{~kg}$.

Fêmeas originadas de cruzamentos com touros Nelore, Guzerá, Red Angus, Marchigiana e Simental e vacas Nelore, via inseminação artificial, eram incorporadas ao rebanho reprodutivo entre 18 e 24 meses de idade, praticamente sem seleção, exceto por inaptidão reprodutiva, defeitos anatômicos ou desenvolvimento ponderal insatisfatório.

Os animais foram submetidos a pastagens cultivadas de $B$. humidicola e $B$. decumbens. No inverno, recebiam suplementação de forragem picada - mistura de capim-elefante ( $P$. pupureum) e cana-de-açúcar ( $S$. cerevisiae), uréia adicionada à mistura mineral, além de pastejo direto em bancos de proteína de leucena (Leucaena spp) e guandu (C. cajan).

Os dados das pesagens foram organizados e tabulados individualmente a partir do peso, ao nascimento, de cada fêmea, bem como das observações de pesagens obtidas com o avançar da idade e nos períodos de gestação e lactação. Consideraram-se a identificação/número do animal (identificador do grupamento genético), Pn (peso ao nascimento), T (idade em dias), W (Peso do animal), Tp (dias em gestação) e Tl (dias em lactação).

Para descrever a trajetória do crescimento das fêmeas bovinas nos diferentes grupos genéticos $(\mathrm{N}, \mathrm{G}, \mathrm{R}$, $\mathrm{M}$ e $\mathrm{S}$ ), a função proposta $\left(\mathrm{f}_{\text {peso }}\right)$ foi composta pelos componentes: ganhos de peso em função da idade $\left(f_{\text {idade }}\right)$ e com o avançar da gestação ( $f_{\text {gestação }}$, perda de peso no transcorrer da lactação $\left(\mathrm{f}_{\text {lactacão }}\right)$ e erro associado a cada observação $(\varepsilon): \mathrm{f}_{\text {peso }}=\mathrm{f}_{\text {idade }}+\mathrm{f}_{\text {gestação }}+\mathrm{f}_{\text {lactação }}+\varepsilon$.

Para verificar o melhor modelo para descrever o ganho de peso de acordo com a idade, foram ajustadas as seguintes funções: Brody, $\mathrm{f}_{\text {idade }}=\mathrm{Pn}+\mathrm{A}\left(1-\mathrm{Be}^{-\mathrm{Kt}}\right)$; Von Bertalanffy,

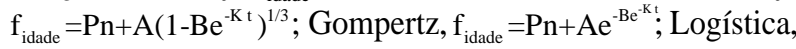
$\mathrm{f}_{\text {idade }}=\mathrm{Pn}+\mathrm{A}\left(1+\mathrm{Be}^{-\mathrm{Kt}}\right)^{-1}$, e Michaelis-Menten reparametrizada, 
$\mathrm{f}_{\text {idade }}=\frac{\left(\mathrm{PnK}^{\mathrm{C}}+\mathrm{At}^{\mathrm{C}}\right)}{\left(\mathrm{K}^{\mathrm{C}}+\mathrm{t}^{\mathrm{C}}\right)} \cdot$ Em que: $\mathrm{f}$ (idade) é o peso corporal à idade t (dias); Pn é o peso corporal ao nascer (kg); A é o peso assintótico quando t tende a $+\infty$ (interpretado como peso à idade adulta); B é uma constante de integração sem interpretação biológica bem definida. O parâmetro $\mathrm{C}$ é uma constante positiva e $\mathrm{K}$ é interpretado como taxa de crescimento (deve ser entendida como mudança de peso em relação ao peso à maturidade).

$\mathrm{O}$ efeito da gestação $\mathrm{f}_{\text {gestação }}$ no peso de uma vaca é somente visível após 50 dias (Korver et al., 1985). Portanto, para descrever o ganho de peso de acordo com a gestação, foi usada uma função quadrática $\mathrm{f}_{\text {gestação }}=\mathrm{Gpg}_{\max } *\left(\frac{\mathrm{Dg}-50}{\mathrm{Dpg}_{\max }}\right) *\left[2-\left(\frac{\mathrm{Dg}-50}{\mathrm{Dpg}_{\max }}\right)\right] ;$ sendo considerado Dg como os dias em gestação; $\mathrm{Gpg}_{\max }$ como o ganho máximo de peso acumulado na gestação; $\mathrm{Dpg}_{\max }$ o dia de ocorrência do ganho de peso máximo acumulado na gestação.

Para descrever a perda de peso no decorrer da lactação $f_{\text {lactação }}$ utilizou-se a função de Wood reparametrizada (Wood, 1967), $\mathrm{f}_{\text {lactą̧ăo }}=\mathrm{Ppl}_{\max } *\left(\frac{\mathrm{Dl}}{\mathrm{DPpl}_{\text {max }}}\right)^{\mathrm{s}} * \operatorname{exps} *\left(1-\frac{\mathrm{Dl}}{\mathrm{DPpl}} \mathrm{max}_{\text {ax }}\right)$; sendo considerado $\mathrm{Dl}$ como os dias em lactação; $\mathrm{Ppl}$ max como o peso máximo acumulado perdido na lactação; $\mathrm{DPpl}_{\max }$ como o dia de ocorrência da máxima perda de peso acumulado na lactação e $\mathrm{s}$ a declividade da curva de lactação.

Os parâmetros das funções foram estimados com o auxílio do software NLREG versão 4.1.

Para selecionar a função que melhor descreve a curva de crescimento, utilizaram-se os critérios: Quadrado Médio do Resíduo (QMR), Coeficiente de Determinação Múltiplo Ajustado $\left(R_{a}^{2}\right.$ ), Desvio Médio Absoluto (DMA) e Análise Gráfica dos Resíduos.

$\mathrm{O} \mathrm{R}_{\mathrm{a}}^{2}$ foi calculado como o quadrado da correlação entre os pesos observados e estimados, equivalente a: $\frac{(\mathrm{n}-1)}{(\mathrm{n}-\mathrm{p})}\left(1-\left(\frac{\mathrm{SQR}}{\mathrm{SQTc}}\right)\right)$, em que $\mathrm{n}$ é o número de observações, $\mathrm{p}$ é o número de parâmetros ajustados, $S Q R$ é a soma de quadrados do resíduo e SQTc a soma de quadrados total corrigida pela média. Quanto mais próximo de 1, melhor o ajuste da curva.

O Desvio Médio Absoluto dos Resíduos (DMA), estatística proposta por Sarmento et al. (2006), para avaliar a qualidade de ajuste para cada amostra, foi calculado como: ${ }_{D M A}=\frac{\sum_{\mathrm{i}=1}^{\mathrm{n}}\left|\mathrm{Y}_{\mathrm{i}}-\hat{\mathrm{Y}}_{\mathrm{i}}\right|}{\mathrm{n}}$, em que $\mathrm{Y}_{\mathrm{i}}$ é o valor do peso observado $\hat{\mathrm{Y}}_{\mathrm{i}}$ o valor do peso estimado e n o tamanho da amostra. Quanto menor o valor do DMA, melhor o ajuste da curva.
A diferença entre os valores de pesos observados e estimados (resíduos) foi ilustrada graficamente a fim de possibilitar a visualização da dispersão dos resíduos.

\section{RESULTADOS E DISCUSSÃO}

Os valores estimados dos critérios utilizados para avaliar o ajuste da função que descreve a curva de crescimento de fêmeas Nelores e Cruzadas em função da idade, da gestação e da lactação encontram-se na Tabela 1.

Evidencia-se a partir dos Coeficientes de Determinação Múltiplo Ajustado $\left(\mathrm{R}_{\mathrm{a}}^{2}\right)$ que, independentemente do grupamento genético $(\mathrm{G}, \mathrm{M}, \mathrm{N}, \mathrm{R}$ e S), ocorreu bom ajuste para todas as funções, variando entre 0,76 e 0,93. Entretanto, as funções que melhor se ajustaram ao peso das fêmeas foram as de MichaelisMenten reparametrizada, Brody e Von Bertalanffy (valores mais próximos de 1).

Ao analisar os Quadrados Médios dos Resíduos (QMR), verificam-se menores valores ao ajustar a função de Michaelis-Menten reparametrizada quando se estudaram os grupamentos G, M, N, R e S, seguido das funções de Brody e Von Bertalanffy, com pequena diferença entre estas. A qualidade do ajuste para as funções de Gompertz e Logística apresentaram os maiores valores de QMR. Esses resultados reforçam o que foi dito anteriormente, com relação ao $R_{a}^{2}$, sobre a qualidade dos ajustes das funções Michaelis-Menten, Brody e Von Bertalanffy.

Dentre as funções estudadas, independentemente do grupamento genético utilizado, os valores observados para os Desvios Médios Absolutos (DMA) obtidos para a função de Michaelis-Menten reparametrizada foram os menores.

Analisando-se as curvas de crescimento (Figuras 1, 2, 3, 4 e 5), respectivamente, para os grupos G, M, N, R e S, pode-se constatar que os desvios obtidos, quando ajustadas as funções de Gompertz e Logística, foram maiores, sendo menores nos ajustes das funções de Michaelis-Menten reparametrizada, Brody e Von Bertalanffy quando comparadas à função dos Pesos Observados. Os ajustes dos três últimos foram semelhantes, alternando-se ao longo da curva de crescimento. Porém, ao serem observadas as dispersões gráficas para a função de Michaelis-Menten reparametrizada, esta, aparentemente, apresentou melhores resultados. Comprovações semelhantes às obtidas quando analisados $R_{a}^{2}$, QMR e DMA. 
Tabela 1 - Quadrado Médio do Resíduo, Coeficiente de Determinação Múltiplo Ajustado e Desvio Médio Absoluto, ao ajustar o crescimento de fêmeas Nelore e seus Cruzamentos em função da idade, gestação e lactação.

\begin{tabular}{|c|c|c|c|}
\hline Modelos & Quadrado Médio do Resíduo & $\begin{array}{c}\text { Coeficiente de Determinação } \\
\text { Múltiplo Ajustado }\end{array}$ & Desvio médio Absoluto \\
\hline \multicolumn{4}{|c|}{ Nelore x Nelore (Grupamento N) } \\
\hline A & 877,36 & 0,87 & 0,41 \\
\hline $\mathrm{B}$ & 655,46 & 0,91 & 0,00 \\
\hline $\mathrm{C}$ & 858,86 & 0,89 & 1,54 \\
\hline $\mathrm{D}$ & 1244,67 & 0,83 & 2,13 \\
\hline $\mathrm{E}$ & 824,52 & 0,90 & 1,43 \\
\hline \multicolumn{4}{|c|}{ Nelore x Marchigiana (Grupamento M) } \\
\hline A & 1086,85 & 0,92 & $-5,89$ \\
\hline $\mathrm{B}$ & 981,05 & 0,91 & 0,00 \\
\hline $\mathrm{C}$ & 1912,26 & 0,86 & 2,07 \\
\hline $\mathrm{D}$ & 3276,05 & 0,76 & 4,25 \\
\hline $\mathrm{E}$ & 1085,87 & 0,91 & 1,03 \\
\hline \multicolumn{4}{|c|}{ Nelore x Guzerá (Grupamento G) } \\
\hline A & 716,27 & 0,90 & $-0,08$ \\
\hline $\mathrm{B}$ & 638,45 & 0,91 & 0,00 \\
\hline $\mathrm{C}$ & 817,72 & 0,88 & 0,79 \\
\hline $\mathrm{D}$ & 1377,74 & 0,82 & 1,01 \\
\hline $\mathrm{E}$ & 688,39 & 0,90 & 0,74 \\
\hline \multicolumn{4}{|c|}{ Nelore x Red-Angus (Grupamento R) } \\
\hline A & 707,78 & 0,92 & 0,6004 \\
\hline $\mathrm{B}$ & 689,37 & 0,93 & 0,0000 \\
\hline $\mathrm{C}$ & 1012,23 & 0,90 & 2,2072 \\
\hline $\mathrm{D}$ & 1877,26 & 0,83 & 2,2167 \\
\hline $\mathrm{E}$ & 953,34 & 0,91 & 1,5451 \\
\hline \multicolumn{4}{|c|}{ Nelore x Simental (Grupamento S) } \\
\hline A & 925,92 & 0,91 & 0,1220 \\
\hline B & 795,8244 & 0,92 & 0,0000 \\
\hline $\mathrm{C}$ & 1006,5662 & 0,89 & 1,2857 \\
\hline $\mathrm{D}$ & 2183,5073 & 0,76 & 3,0117 \\
\hline $\mathrm{E}$ & 1048,5217 & 0,89 & 1,0650 \\
\hline
\end{tabular}

A - Função de Brody; B - Função de Michaelis Menten reparametrizada; C - Função de Gompertz; D - Função de Logística; E Função de Von Bertalanffy.

A função de Michaelis-Menten reparametrizada apresentou os melhores ajustes para descrever o crescimento das fêmeas Nelore e Cruzadas em função da idade, simultaneamente com as funções para o ganho de peso com a gestação e a perda de peso durante a lactação. 

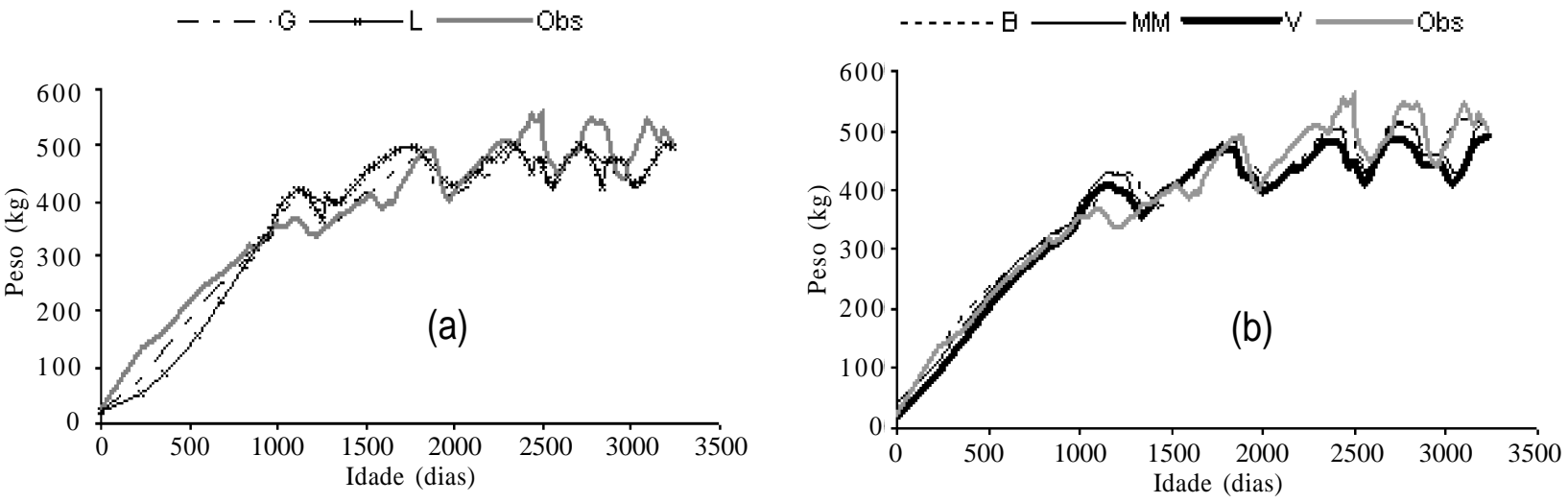

Figura 1 - Funções de Gompertz (G), Logística (L) e dos Pesos Observados (Obs) - (a) e Brody (B), Michaelis-Menten reparametrizada (MM), Von Bertalanffy (V) e dos Pesos Observados (Obs) - (b) de fêmeas do grupamento genético G (F1 Nelore-Guzerá).
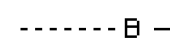

WhI
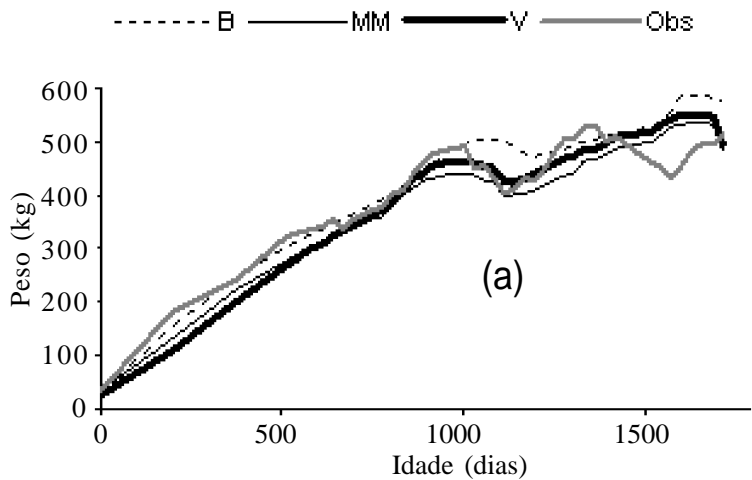
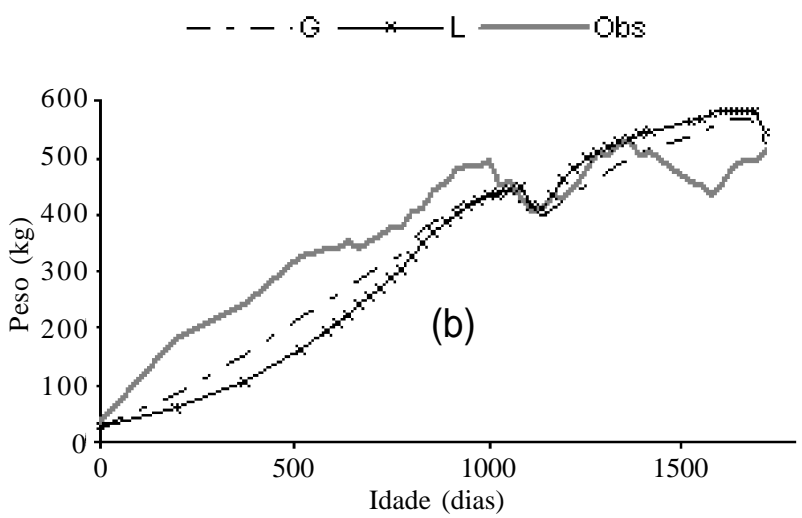

Figura 2 - Funções de Brody (B), Michaelis-Menten reparametrizada (MM), Von Bertalanffy (V) e dos Pesos Observados (Obs) - (a) e das funções de Gompertz (G), Logística (L) e dos Pesos Observados (Obs) - (b) de fêmeas do grupamento genético M (F1 Nelore-Marchigiana).

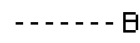

$\mathrm{B}$

MM

$\gamma$

Obs

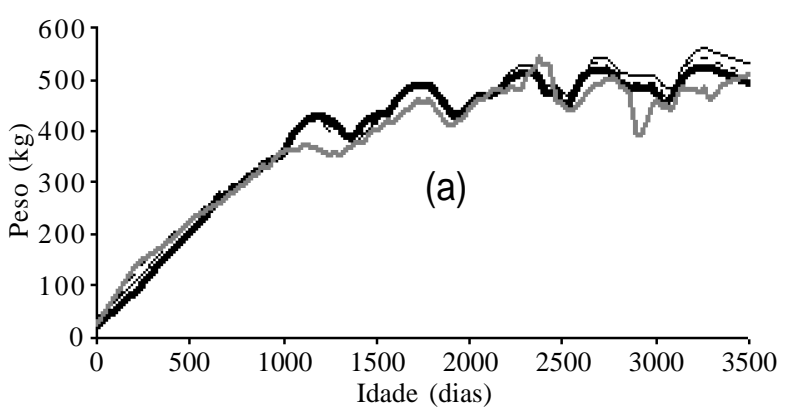

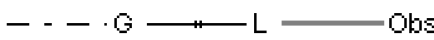

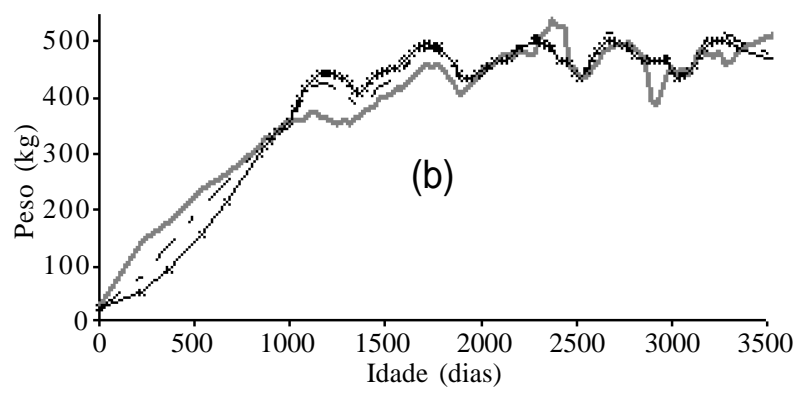

Figura 3 - Funções de Brody (B), Michaelis-Menten reparametrizada (MM), Von Bertalanffy (V) e dos Pesos Observados (Obs) - (a) e das funções de Gompertz (G), Logística (L) e dos Pesos Observados - (b) de fêmeas do grupamento genético N (F1 Nelore- Nelore 1). 

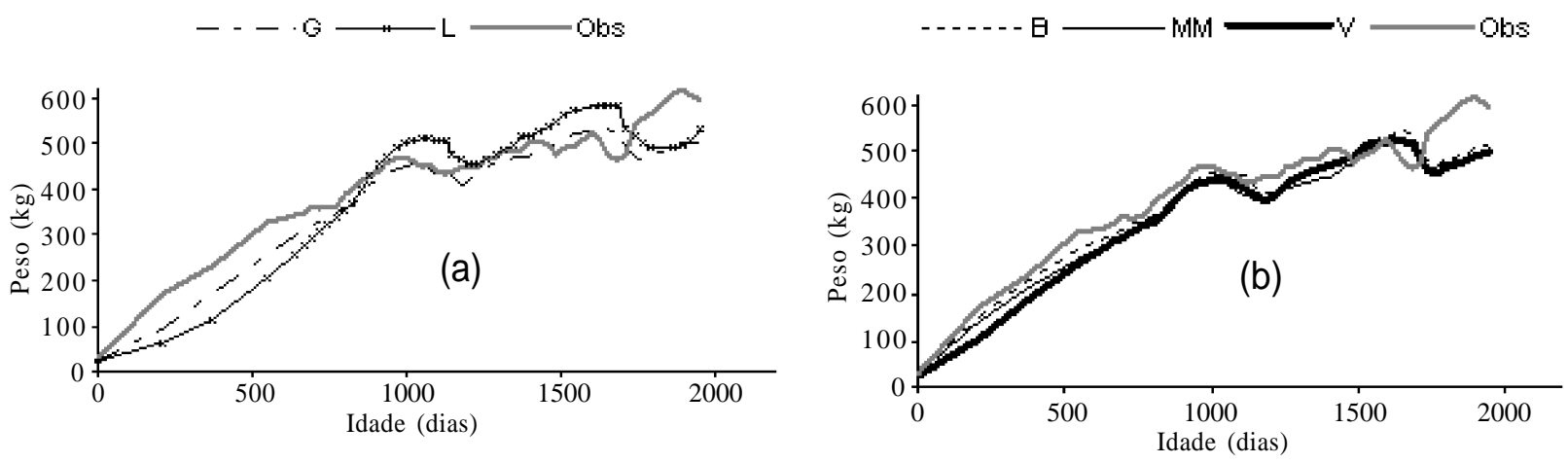

Figura 4 - Funções de Gompertz (G), Logística (L) e dos Pesos Observados (Obs) - (a) e de Brody (B), MichaelisMenten reparametrizada (MM), Von Bertalanffy (V) e dos Pesos Observados (Obs) - (b) de fêmeas do grupamento genético R (F1 Nelore-Red-Angus).
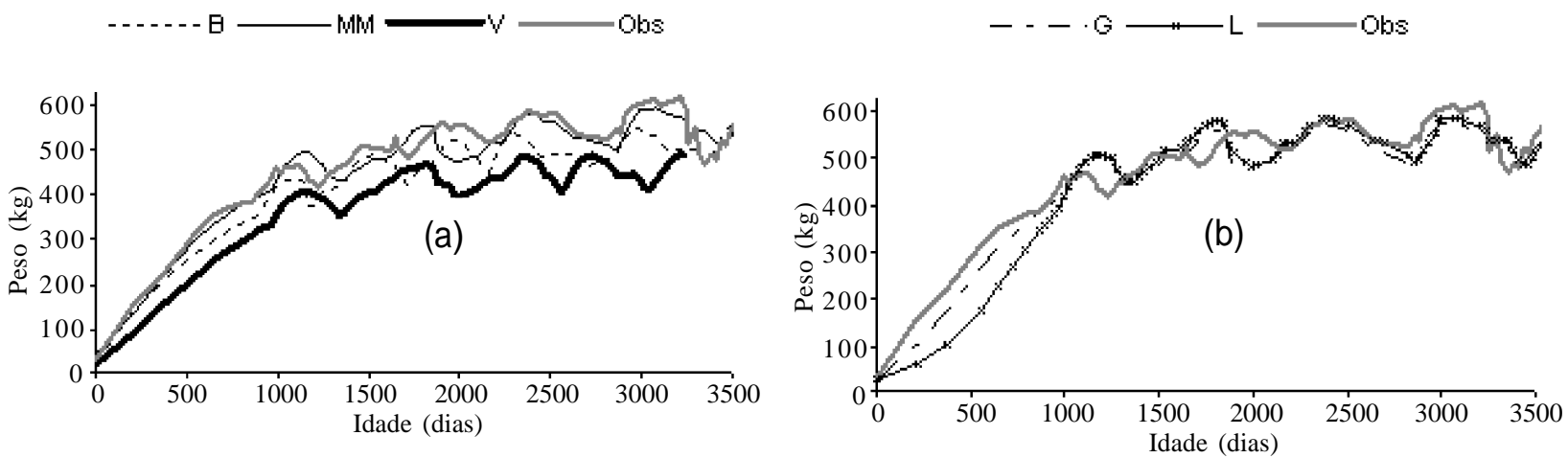

Figura 5 - Funções de Brody (B), Michaelis-Menten reparametrizada (MM), Von Bertalanffy (V) e dos Pesos Observados (Obs) - (a) e das funções de Gompertz (G) e Logística (L) - (b) e dos Pesos Observados (Obs) de fêmeas do grupamento genético S (F1 Nelore-Simental).

Ao se avaliar as estimativas dos parâmetros (Tabela 2), verifica-se que o peso ao nascer estimado (Pn) apresentou para o grupo genético $\mathrm{N}$ valor de $40,71 \mathrm{~kg}$, superior ao encontrado por Mariante et al. (1985) de 31,88 $\mathrm{kg}$; Eller et al. (1989) de 30,45 kg, para a raça Nelore. Segundo Pelicioni et al. (2003), as médias de peso ao nascimento para a raça Guzerá foi de $29,74 \mathrm{~kg}$, inferior ao encontrado no cruzamento Guzerá x Nelore $(43,59 \mathrm{~kg})$ no presente trabalho. Nos cruzamentos com as raças taurinas Red-Angus, Marchigiana e Simental, os valores de Pn ( $\mathrm{R}=$ $30,11 \mathrm{~kg}, \mathrm{M}=36,81 \mathrm{~kg}$ e $\mathrm{S}=44,04 \mathrm{~kg}$ ) se aproximam daqueles encontrados na literatura.

Verifica-se que os animais dos cruzamentos com Red-Angus e Marchigiana apresentaram maior peso adulto (A), acima de $650 \mathrm{~kg}$, se comparado aos cruzamentos com zebuínos. Os animais do grupamento $\mathrm{S}$ obtiveram peso adulto de $568,78 \mathrm{~kg}$, semelhante ao grupamento $\mathrm{N}$ $(577,70 \mathrm{~kg})$.
A estimativa do parâmetro C (Tabela 2), que está diretamente relacionado com o ponto de inflexão, foi superior a 1, de modo que todas as curvas foram sigmóides devido à existência de um ponto de inflexão. Este parâmetro pode ser interpretado como taxa de maturação, devendo ser entendida como a mudança de peso em relação ao peso na maturidade, ou seja, indicador da idade em que o animal se aproxima do seu tamanho adulto. Animais com maior valor para parâmetro $\mathrm{C}$ possuem menor peso adulto (A).

Os animais do grupamento genético $\mathrm{G}$ apresentaram uma maior taxa de crescimento (c), sendo os mais precoces, enquanto os animais do grupamento $\mathrm{R}$ foram os mais tardios.

$\mathrm{O}$ valor do parâmetro $\mathrm{K}$ (tempo em que os animais atingem metade do seu peso adulto) foi maior no grupo $\mathrm{R}$ e menor no grupo $\mathrm{S}$, o que não indica que os animais do grupo R sejam mais tardios que os do grupamento $\mathrm{S}$, pois estes atingem diferentes tamanhos à maturidade. 
Tabela 2 - Estimativas dos parâmetros (C, K, Pn, A, B, Gpg, Dpg, Ppl, DPpl, S)*, ao ajustar os modelos: A - Função de Brody; B - Função de Michaelis Menten reparametrizada; C - Função de Gompertz; D - Função de Logística; E - Função de Von Bertalanffy para os diferentes grupamentos genéticos.

\begin{tabular}{|c|c|c|c|c|c|c|c|c|c|c|}
\hline Modelo & $\mathrm{c}$ & $\mathrm{K}$ & Pn & A & B & Gpg & Dpg & Ppl & DPpI & $\mathrm{S}$ \\
\hline \multicolumn{11}{|c|}{ Nelore - Nelore (Grupamento N) } \\
\hline A & - & - & 25,644 & 513,473 & 0,001 & 42,371 & 189,972 & $-39,077$ & 92,856 & 8,943 \\
\hline B & 1,459 & 787,979 & 40,712 & 577,702 & - & 42,736 & 194,186 & $-30,714$ & 102,757 & 3,353 \\
\hline $\mathrm{C}$ & - & - & 23,779 & 475,580 & 0,002 & 41,337 & 194,612 & $-32,902$ & 83,105 & 2,881 \\
\hline $\mathrm{D}$ & - & - & 23,218 & 464,353 & 0,004 & 35,920 & 197,704 & $-29,518$ & 72,402 & 3,052 \\
\hline $\mathrm{E}$ & - & - & 24,294 & 485,871 & 0,002 & 39,512 & 200,537 & $-32,635$ & 77,368 & 4,680 \\
\hline \multicolumn{11}{|c|}{ Nelore - Marchigiana (Grupamento M) } \\
\hline A & - & - & 30,841 & 616,822 & 0,001 & 51,411 & 200,881 & $-10,005$ & 48,350 & 0,889 \\
\hline B & 1,289 & 726,648 & 36,813 & 657,429 & - & 40,023 & 189,856 & $-37,172$ & 62,928 & 0,661 \\
\hline $\mathrm{C}$ & - & - & 29,821 & 596,418 & 0,002 & 31,549 & 183,306 & $-50,172$ & 53,917 & 1,000 \\
\hline $\mathrm{D}$ & - & - & 28,900 & 577,996 & 0,004 & 22,096 & 181,090 & $-62,503$ & 47,515 & 1,511 \\
\hline $\mathrm{E}$ & - & - & 26,937 & 538,729 & 0,002 & 40,754 & 192,447 & $-33,639$ & 51,046 & 0,857 \\
\hline \multicolumn{11}{|c|}{ Nelore - Guzerá (Grupamento G) } \\
\hline A & - & - & 24,144 & 482,886 & 0,001 & 48,032 & 215,346 & $-35,818$ & 84,902 & 6,049 \\
\hline B & 1,7353 & 612,5056 & 43,585 & 488,330 & - & 52,291 & 227,160 & $-34,944$ & 109,946 & 6,824 \\
\hline $\mathrm{C}$ & - & - & 22,747 & 454,944 & 0,002 & 43,127 & 202,590 & $-30,567$ & 75,781 & 3,099 \\
\hline $\mathrm{D}$ & - & - & 23,782 & 475,636 & 0,004 & 28,145 & 151,795 & $-43,816$ & 74,953 & 1,500 \\
\hline $\mathrm{E}$ & - & - & 22,383 & 447,658 & 0,002 & 43,447 & 202,572 & $-35,341$ & 86,847 & 5,146 \\
\hline \multicolumn{11}{|c|}{ Nelore - Red-angus (Grupamento R) } \\
\hline A & - & - & 28,222 & 564,448 & 0,0012 & 46,441 & 206,691 & $-32,1252$ & 68,505 & 4,027 \\
\hline B & 1,1431 & 854,390 & 30,113 & 679,681 & - & 51,919 & 190,295 & $-24,3397$ & 45,971 & 1,324 \\
\hline $\mathrm{C}$ & - & - & 25,712 & 514,233 & 0,003 & 41,037 & 184,028 & $-37,4434$ & 58,809 & 3,477 \\
\hline $\mathrm{D}$ & - & - & 26,657 & 533,132 & 0,0045 & 59,5198 & 210,026 & $-42,1225$ & 107,798 & 2,793 \\
\hline $\mathrm{E}$ & - & - & 26,013 & 520,258 & 0,0021 & 38,1987 & 182,019 & $-42,6012$ & 60,349 & 1,987 \\
\hline \multicolumn{11}{|c|}{ Nelore - SimentaI (Grupamento S) } \\
\hline A & - & - & 25,338 & 506,770 & 0,001 & 51,997 & 190,916 & $-32,315$ & 63,345 & 5,817 \\
\hline B & 1,5929 & 567,004 & 44,041 & 568,784 & - & 56,246 & 201,958 & $-30,938$ & 92,395 & 2,873 \\
\hline $\mathrm{C}$ & - & - & 26,206 & 524,124 & 0,003 & 50,125 & 209,711 & $-36,658$ & 71,162 & 2,439 \\
\hline $\mathrm{D}$ & - & - & 26,749 & 534,976 & 0,004 & 48,324 & 207,440 & $-49,697$ & 101,600 & 2,644 \\
\hline $\mathrm{E}$ & - & - & 26,277 & 525,530 & 0,002 & 53,967 & 206,303 & $-33,195$ & 65,352 & 2,334 \\
\hline
\end{tabular}

$\mathrm{C}=$ taxa de crescimento; $\mathrm{K}=$ metade do tempo em que o animal atinge seu crescimento máximo; $\mathrm{Pn}=$ peso ao nascer predito; $\mathrm{A}=$ peso assintótico $; \mathrm{B}=$ constante relacionada com peso ao nascimento, $\mathrm{Gpg}=$ ganho de peso máximo acumulado durante a gestação; Dpg = dia em que ocorre o máximo ganho de peso acumulado durante a gestação; Ppl = perda de peso máxima acumulada durante a lactação; $\mathrm{DPpl}=$ dia em que ocorre a máxima perda de peso acumulada durante a lactação; $\mathrm{S}=$ declividade da curva. 
A estimativa do ganho máximo de peso durante a gestação (Gpg) (Tabela 2) foi maior para os grupamentos G, R e S $(52,29 \mathrm{~kg}, 51,92 \mathrm{~kg}$ e $56,25 \mathrm{~kg}$, respectivamente), o que pode ser atribuído a fatores como a diferença entre o tamanho corporal dos animais que compõem os grupamentos genéticos em questão e, possivelmente, devido a diferenças metabólicas e de adaptação ao ambiente da região. A média do Gpg dos grupamentos com fêmeas Nelores e Cruzadas com zebuínos foi de $47,51 \mathrm{~kg}$ e nas fêmeas oriundas de cruzamentos com taurinos foi de $49,39 \mathrm{~kg}$. A média do Gpg, considerando os cinco grupamentos genéticos neste estudo, foi de $48,45 \mathrm{~kg}$, resultado inferior aos valores médios obtidos por Bereskin \& Touchberry (1967) que, ao analisarem informações de vacas das raças leiteiras Guernsey, Holandesas e mestiças 11/16, 8/16 e 5/16 Holandês, abatidas em estágios sucessivos de prenhez, encontraram ganho de peso total durante a gestação de $65 \mathrm{~kg}$.

A média dos dias em que ocorre o máximo ganho de peso acumulado na gestação (Dpg) para os grupamentos foi de 200 dias, período em que é possível observar o máximo desenvolvimento do feto, ou seja, no terço final da gestação. Portanto, independente do grupamento genético estudado, é necessária uma maior atenção no terço final da gestação.

As estimativas do parâmetro relacionado com a perda de peso máximo acumulado durante a lactação $(\mathrm{Ppl})$ evidenciam (Tabela 2) uma superioridade do grupamento genético $\mathrm{R}$ em relação aos demais, ou seja, os animais do grupamento $\mathrm{R}$ apresentaram menores valores de $\mathrm{Ppl}$ $(24,40) \mathrm{kg}$. Isso pode ter ocorrido devido ao fato de os animais do grupamento $\mathrm{R}$ terem produzido menos leite devido a menor persistência na lactação.

Quanto ao dia em que ocorre a máxima perda de peso acumulada durante a lactação (DPpl) constata-se (Tabela 2) que, no grupamento genético $\mathrm{G}$ a DPpl, ocorreu mais tardiamente (109 dias), seguido pelos grupamentos N (102), S (92),M (62) e R (46), devendo estar relacionado a um pico de lactação mais tardio para os animais destes grupamentos. Isso pode ser justificado com a máxima perda de peso que estes animais obtiveram coincidindo com o máximo desbalanço energético.

Com base nos valores do parâmetro $\mathrm{S}$ (relacionado com a declividade da curva de lactação), observa-se (Tabela 2) que quanto maior o seu valor, menor será a persistência da curva de lactação com a possibilidade de uma menor produção de leite. Isso implica menores perdas de peso acumulado durante a lactação, como visto na discussão sobre o parâmetro Ppl. Um baixo valor do parâmetro $\mathrm{S}$ favorece o desenvolvimento de crias mais pesadas, pois pode possibilitar a amamentação por um maior período.

\section{CONCLUSÕES}

Dentre as funções, a de Michaelis-Menten reparametrizada apresentou a melhor descrição e interpretação biológica do crescimento de fêmeas Nelore e Cruzadas, levando em consideração os ajustes para os efeitos da gestação e lactação.

Fica evidente a necessidade de ajuste de funções diferenciadas para fêmeas, bem como estudos com um maior número de vacas para confirmar os resultados obtidos neste trabalho.

\section{REFERÊNCIAS BIBLIOGRÁFICAS}

BERESKIN, B.; TOUCHBERRY, R.W. Some effects of pregnancy on body weight and paunch girth. Journal of Dairy Science, Urbana, v.50, n.2, 1967.

\section{BRODY, S. Bioenergetics and growth. New York:} Reinhold, 1945. 1023p.

ELLER, J.P.; LÔBO, R.B.; ROSA, A.N. Influência de fatores genéticos e de meio em pesos de bovinos da raça Nelore criados no Estado de São Paulo. Revista da Sociedade Brasileira de Zootecnia, Viçosa, v.18, n.2, p.103-111, 1989.

GARNERO, A.V.; MARCONDES, C.R.; BEZERRA, L.A.F.; OLIVEIRA, H.N.; LÔBO, R.B. Parâmetros genéticos da taxa de maturação e do peso assintótico de fêmeas da raça Nelore. Arquivo Brasileiro Medicina

Veterinária e Zootecnia, Belo Horizonte, v.57, n.5, p.652662, 2005.

KORVER, S.; ARENDONK, J.A.M. van; KOOPS, W.J. A function for live weight change between two calvings in dairy cattle. Animal Production, v.40, p.233-241, 1985.

LAMBE, N.R.; NAVAJAS, E.A.; SIMM, G.; BÜNGER, L. A genetic investigation of various growth models to describe growth of lambs of two contrasting breeds. Journal of Animal Science, v.84, p.2642-2654, 2006.

LÓPEZ, S.; FRANCE, J.; GERRITS, W.J.J.; DHANOA, M.S.; HUMPHRIES, D.J.; DIJKSTRA, J. A generalized Michaelis-Menten equation for the analyses of growth. Journal of Animal Science, v.78, p.1816-1828, 2000. 
MARIANTE, A.S.; NOBRE, P.R.C.; ROSA, A.N.; EVANGELISTA, S.R.M. Resultados do controle de desenvolvimento ponderal: raça Nelore 1975-1984. Campo Grande: Embrapa-CNPGC, 1985. 88p.

(Documentos, 25).

PELICIONI, L.C.; QUEIROZ, S.A.; ALBUQUERQUE, L.G. Estimativas de parâmetros genéticos para pesos ao nascer e mensais até 450 dias em bovinos Guzerá.

Archives Latinoamericano de Prodution Animal, v.11, n.1, p.34-39, 2003 .
RICHARDS, F.J. A flexible growth function for empirical use. Journal of Experimental Botany, v.10, p.290-300, 1959.

SARMENTO, J.L.R.; REGAZZI, A.J.; SOUSA, W.H.; TORRES, R.A.; BREDA, F.C.; MENEZES, G.R.O. Estudo da curva de crescimento de ovinos Santa Inês. Revista Brasileira de Zootecnia, Viçosa, v.35, n.2, p.435-442, 2006.

WOOD, P.D.P. Algebric model of lactation curve in cattle. Nature, London, v.216, n.5111, p.164-165, 1967. 